\title{
0 impacto da estratégia saúde da família nos indicadores de saúde bucal
}

\author{
Thiago de Carvalho REIS'; Igor Henrique Teixeira FUMAGALLI®;

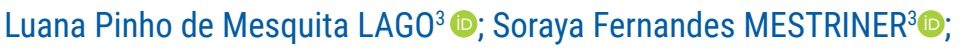 \\ Marta Moraes RAMOS4; Wilson MESTRINER JÚNIOR ${ }^{5}$ [0
}

1 - Mestrando do Programa de Diferenciação Celular Normal e Neoplásica - Faculdade de Medicina de Ribeirão Preto da Universidade de São Paulo; 2 - Mestrando do Programa de Pós-Graduação em Saúde Pública da Escola de Enfermagem de Ribeirão Preto da Universidade de São Paulo; 3 - Professora Doutora da Faculdade de Odontologia de Ribeirão Preto, Departamento de Estomatologia, Saúde Coletiva e Odontologia Legal da Universidade de São Paulo; 4 - Articuladora da Atenção Básica do Departamento Regional de Saúde XIII - Ribeirão Preto; 5 - Professor Titular da Faculdade de Odontologia de Ribeirão Preto, Departamento de Estomatologia, Saúde Coletiva e Odontologia Legal da Universidade de São Paulo.

\section{Resumo}

Objetivo: avaliar nos 90 municípios da Rede Regional de Atenção à Saúde 13 (RRAS 13) o impacto das coberturas da Estratégia de Saúde da Família sobre os indicadores de saúde bucal, levando em consideração aspectos socioeconômicos, em uma análise de série histórica entre os anos de 2014 e 2017. Material e método: Utilizando-se do Sistema de Informações Ambulatoriais, plataformas eGestor e DATASUS, foram obtidas as variáveis de cobertura populacional pelos serviços de saúde e calculados os indicadores de saúde bucal. Aspectos socioeconômicos foram considerados usando ponderamento pelo IDH municipal, seus componentes de renda, escolaridade e índice de Gini. Foi realizada uma análise descritiva dos dados e posteriormente proposto uma modelagem bayesiana espaço temporal usando-se modelo espacial de Poisson com efeito aleatório BYM. Os indicadores sociais entraram como preditores no modelo, que foi implementado com apoio computacional do software $\mathrm{R}$ e utilizou métodos de aproximação de Laplace. Resultados: Apontaram que existe evidência de relação estatística das coberturas de saúde bucal na Estratégia de Saúde da Família com melhora dos indicadores "proporção de exodontia em relação aos procedimentos" e "cobertura de primeira consulta odontológica programática”. Já as coberturas de saúde bucal no modelo tradicional se relacionaram estatisticamente com piora destes dois indicadores. Conclusão: Maior cobertura por equipes de saúde bucal na ESF foi associada à melhora dos indicadores de saúde bucal avaliados neste trabalho, quando comparado ao modelo tradicional.

PALAVRAS-CHAVE: Indicadores Básicos de Saúde; Avaliação do Impacto na Saúde; Atenção Primária à Saúde; Estratégia Saúde da Família; Saúde Bucal.

Copyright $(\odot 2021$ Revista Odontológica do Brasil Centra Esta obra está licenciada com uma licença Atribuição-NãoComerciaCompartilhalgual 4.0 Internaciona (CCBY-NC-SA 4.0)

Recebido: 15/10/20 Aceito: 05/05/21 Publicado: 01/07/21

\section{AUTOR PARA CORRESPONDÊNCIA}

Thiago de Carvalho Reis

Rua Desembargador Edgard de Moura Bittensour, n. 140, Bloco D7, Apt. 1,

Cep.: 14030-400, Bairro: Vila Virgínia, Ribeirão Preto-SP, Brasil

E-mail: thiagodec.reis@gmail.com 


\section{Introdução}

A partir do século XX, a forma de organização das práticas de saúde no Brasil, notadamente influenciada pelo contexto mundial, incorporou o modelo biomédico. Este modelo, que passa a ditar a organização dos serviços de saúde e o tipo de assistência prestado pelos serviços, possui limitações conhecidas como: Ênfase na atenção hospitalar, utilização de tecnologias duras, curativismo e visão do corpo doente como uma máquina. A necessidade de se romper com este modelo, indicou a Estratégia de Saúde da Família (ESF) como a estratégia prioritária para reorientação do modelo de atenção em saúde no Brasil ${ }^{1}$.

A ESF surge em 1994, em 2018 contava com 43.454 equipes com uma cobertura de $64,76 \%$ da população brasileira; e cobertura de saúde bucal na ESF de 28.096 equipes com 42,23\% de cobertura (dados de novembro de 2018)2 ${ }^{2}$ É inquestionável que tamanho investimento do Ministério da Saúde, estados e municípios na implantação da ESF requer monitoramento, pois se espera que a reorientação do modelo seja capaz de causar impacto positivo nos indicadores e, consequentemente, na condição de saúde da população. Um dos recursos para monitoramento é realizá-lo por meio dos Sistemas de Informação em Saúde (SIS), que são importantes ferramentas para a avaliação, acompanhamento e proposição de políticas públicas. Através dos dados coletados nestes sistemas de informações é possível calcular indicadores de saúde que vistos em conjunto, devem refletir a situação sanitária de uma população e servir para a vigilância das condições de saúde ${ }^{1}$.

O processo de avaliação e monitoramento não deve levar em conta somente os indicadores de saúde, mas também indicadores socioeconômicos ${ }^{3-5}$. O próprio Programa de Melhoria do Acesso e da Qualidade da Atenção Básica (PMAQ-AB) em seu segundo ciclo, com o intuito de assegurar maior equidade na comparação das equipes de atenção básica no processo de certificação, criou estratos municipais que levam em conta aspectos sociais, econômicos e demográficos ${ }^{6}$. A literatura apresenta 
indicadores determinantes de desigualdade consolidados que devem ser considerados nos processos avaliativos em saúde, uma vez que os aspectos socioeconômicos são determinantes na condição de saúde. Dentre estes indicadores destaca-se aqui o Índice de Desenvolvimento Humano Municipal (IDH-M), que é formado pelos componentes educação, longevidade e renda, e o Índice de Gini que avalia a concentração de renda de acordo com a população em situação de pobreza e extrema pobreza, de forma que este coeficiente sendo igual a 0 significa ausência de desigualdade e igual a 1 desigualdade máxima ${ }^{7}$.

Apesar de todo o esforço na consolidação da ESF, os estudos publicados na literatura são conflitantes em responder à pergunta: Maior cobertura pela ESF está relacionada à melhora de indicadores de saúde bucal? Somando-se a isso, poucos estudos atuais avaliam, em série histórica, o impacto que as equipes de saúde bucal da estratégia de saúde da família (ESB/ESF) exercem sobre estes indicadores, em comparação com as equipes de saúde bucal no modelo tradicional (ESB/tradicional).

Desta forma, o objetivo geral deste trabalho é verificar se o modelo de atenção ESF foi indutor na melhora dos indicadores de saúde bucal e, consequentemente, na condição de saúde bucal da população, levando em consideração aspectos socioeconômicos. Secundariamente, o presente trabalho objetiva avaliar a evolução temporal das coberturas e indicadores de saúde bucal sob a ótica de estratos municipais.

\section{Material e método}

Trata-se de um estudo ecológico no qual a variável de cobertura das populações abrangidas pelas equipes saúde bucal, seja na ESF seja no modelo assistencial tradicional, foram coletadas e os indicadores de saúde bucal calculados. Os dados foram organizados em série histórica de 2014 a 2017. O universo de estudo foi constituído pelos 90 municípios pertencentes à Rede Regional de Atenção à Saúde 13 (RRAS 13). Tais municípios estão 
localizados na região Nordeste do estado de São Paulo, pertencentes aos Departamentos Regionais de Saúde de Araraquara, Ribeirão Preto, Franca e Barretos.

\section{Coberturas populacionais}

Através da plataforma eGestor - Atenção Básica, as variáveis independentes do estudo foram obtidas. São elas:

a. Porcentagem de cobertura populacional estimada pelas equipes básicas de saúde bucal (cob. pop. SB): Dada pelo percentual da população coberta por equipes de Saúde Bucal da Estratégia Saúde da Família e por equipes de Saúde Bucal equivalentes e parametrizadas na Atenção Básica tradicional em relação à estimativa populacional.

b. Porcentagem de cobertura populacional estimada pelas equipes básicas de saúde bucal na Estratégia de Saúde da Família (cob. pop. SB/ESF): Dada pelo percentual da população coberta pelas equipes de saúde bucal da ESF em relação à estimativa populacional.

c. Porcentagem de cobertura populacional estimada pelas equipes básicas de saúde bucal no modelo tradicional (cob. pop. SB trad.): Esta cobertura foi calculada a partir da subtração das coberturas “cob. pop. SB” e "cob. pop. SB/ESF”. Desta forma obtém-se a cobertura dos demais modelos assistenciais "não ESF”.

\section{Indicadores de saúde bucal}

As variáveis dependentes são os indicadores de saúde bucal os quais foram escolhidos por serem amplamente utilizados na literatura e preconizados pelo Ministério da Saúde, fazendo parte de diferentes pacotes de indicadores: primeiro, segundo e terceiro ciclo do Programa Nacional de Melhoria do Acesso e da Qualidade da Atenção Básica (PMAQ-AB) e Contrato Organizativo de Ação Pública em Saúde (COAP) em sua pactuação de 2013-2015, 2016 e 2017-2021. Além disso, são 
reprodutíveis devido à possibilidade de obtenção dos dados necessários para o cálculo. Os indicadores de saúde bucal utilizados neste estudo foram:

a. Proporção de exodontia em relação aos procedimentos (prop. exo/proc.): Razão entre o número de exodontias e de procedimentos odontológicos individuais preventivos e curativos.

b. Média da ação coletiva de escovação dental supervisionada (média escov. sup.): Razão entre o número médio mensal de residentes que participaram de ação coletiva de escovação dental supervisionada no ano e a população do município.

c. Cobertura de primeira consulta odontológica programática (cob. 1 cons.): É o percentual de pessoas que receberam uma primeira consulta odontológica programática.

Tais indicadores foram calculadas através de dados do Sistema de Informações Ambulatoriais do SUS (SIA-SUS) disponibilizados pelo Departamento de Informática do SUS (DATASUS). O método de cálculo utilizado é o preconizado pelo MS para o primeiro e segundo ciclo do PMAQ e COAP em sua pactuação de 2013 a $2015^{8-9}$ (Quadro 1).

\section{Variáveis socioeconômicas}

As variáveis socioeconômicas, componentes renda e educação do Índice de Desenvolvimento Humano municipal(IDH-M) e Índice de Gini, foram obtidas através do Atlas de desenvolvimento Humano no $\mathrm{Brasil}^{7}$, os dados são relativos ao último censo. Estas variáveis entraram no modelo estatístico como confundidoras.

\section{Estratos Municipais}

Para a interpretação dos dados, os municípios foram classificados em estratos conforme utilizado no segundo ciclo do Programa Nacional de Melhoria do Acesso e da Qualidade da Atenção Básica (PMAQ). A classificação de cada município foi obtida pela plataforma do PMAQ segundo ciclo. Tal classificação leva em conta aspectos sociais, econômicos e demográficos, 
através da construção de um índice (variando de 0 a 10) baseado nos seguintes indicadores: Produto Interno Bruto (PIB) per Capita (peso 2), percentual da população com plano de saúde (peso 1), percentual da população com Bolsa Família (peso 1), percentual da população em extrema pobreza (peso 1), Densidade demográfica (peso 1). Os municípios foram distribuídos em seis estratos, de acordo com a sua pontuação total e seu porte populacional6 ${ }^{6}$ Quadro 2).

Todos os dados foram estruturados em planilhas eletrônicas, criando-se um banco de dados como o apoio do software Microsoft Excel ${ }^{\circledR}$ (2007). Foi realizada conferência entre os indicadores calculados e os indicadores disponíveis no DATASUS.

QUADRO 1 - Método utilizado para cálculos dos indicadores

\section{Indicador Método de cálculo}

Códigos SIA: 0414020138 Exodontia de dentes permanente e 0414020146 Exodontia múltipla com alveoloplastia por sextante $\times 100$

Códigos SIA: 0101020058 Aplicação de Cariostático (por dente); 0101020066 Aplicação de Selante (por dente); 0101020074 Aplicação tópica de flúor (individual por sessão); 0101020090 Selamento provisório de cavidade dentaria; 0307010015 Capeamento pulpar; 0307010031 Restauração de dente permanente anterior; 0307010040 Restauração de dente permanente posterior; 0307020010 Acesso à polpa dentária e medicação (por dente); 0307020029 Curativo de demora c/ ou s/ preparo biomecânico; 0307020037 Obturação de dente decíduo; 0307020045 Obturação em dente permanente birradicular; 0307020053 Obturação em dente permanente c/ três ou mais raízes; 0307020061 Obturação em dente permanente unirradicular; 0307020070

Prop. Exo/Proc Pulpotomia dentária; 0307020088 Retratamento endodôntico em dente permanente birradicular; 0307020096 Retratamento endodôntico em dente permanente c/ 3 ou mais raízes; 0307020100 Retratamento endodôntico em dente permanente unirradicular; 0307020118 Selamento de perfuração radicular;0307030016 Raspagem alisamento e polimento supragengivais (por sextante) ; 0307030040 Profilaxia/remoção da placa bacteriana; 0307030059 Raspagem alisamento e polimento supragengivais (por sextante); 0307030024 Raspagem alisamento subgengivais (por sextante); 0307030032 Raspagem coronorradicular (por sextante); 0414020022 Apicectomia com ou sem obturação retrógrada; 0414020073 Curetagem periapical; 0414020138 Exodontia de dente permanente; 0414020146 Exodontia Múltipla com alveoloplastia por sextante; 0414020154 Gengivectomia (por sextante); 0414020162 Gengivoplastia (por sextante); 0414020219 Odontossecção/ Radilectomia/Tunelização; 0414020243 Reimplante e transplante dental (por elemento); 0414020367 Tratamento cirúrgico para tracionamento dental; 0414020375 Tratamento cirúrgico periodontal (por sextante). 
QUADRO 2 - Estratos municipais conforme preconizado pelo PMAQ-AB

\section{Estratos Características}

1 Municípios com pontuação menor que 4,82 e população de até 10 mil habitantes

2 Municípios com pontuação menor que 4,82 e população de até 20 mil habitantes

3 Municípios com pontuação menor que 4,82 e população de até 50 mil habitantes.

Municípios com pontuação entre 4,82 e 5,4 e população de até 100 mil habitantes; e municípios com pontuação menor que 4,82 e população entre 50 e 100 mil habitantes.

5 Municípios com pontuação entre 5,4 e 5,85 e população de até 500 mil habitantes; e municípios com pontuação menor que 5,4 e população entre 100 e 500 mil habitantes.

6 Municípios com população acima de 500 mil habitantes ou com pontuação igual ou superior a 5,85

PMAQ-AB: Programa de melhoria de acesso e qualidade da atenção básica ${ }^{6}$

Verificou-se consistência entre os dados. Foram excluídos valores extremos, denominados outliers, que podem ser resultado de possíveis erros no registro das informações e ainda contabilizadas as ocorrências em que os dados não estavam disponíveis no sistema de informação.

\section{Análise estatística}

Utilizou-se de uma análise descritiva das informações coletadas, utilizando-se de medidas descritivas como média, desvio padrão, mínimo, mediana e máximo. Neste cenário de análise, foi proposta uma modelagem bayesiana espaço temporal ${ }^{10}$, usando-se modelo espacial de Poisson com efeito aleatório BYM ${ }^{11}$, onde modelou-se a dependência espacial com matriz de adjacência de ordem 1 que representa a localização de cada município pertencente a RAS13 e seus respectivos vizinhos de primeira ordem, e o padrão temporal relativos aos anos em estudos (2014 a 2017) foi incorporado na modelagem.

Os indicadores sociais como: IDH-M; IDH-Educação; IDHRenda e Índice de Gini entraram como preditores no modelo de forma individual para evitar problemas de colinearidade. $\mathrm{O}$ modelo foi implementado com apoio computacional do software R e utilizou métodos de aproximação de Laplace (INLA) ${ }^{12,13}$. 


\section{Resultados}

Todos os dados referentes às coberturas foram obtidos, sem perdas. Não foi possível calcular por falta de dados informados: $13,61 \%$ das informações do indicador "média da ação coletiva de escovação dental supervisionada"; $1,11 \%$ da "cobertura de primeira consulta odontológica programática" e 1,38\% da "proporção de exodontia em relação aos procedimentos”. Foram considerados outliers 3,61\% das informações e, portanto, excluídos das análises. A distribuição dos municípios estudados por estrato ficou da seguinte forma: 6 municípios no estrato 1, 1 município no estrato 2, 41 municípios no estrato 4, 29 municípios no estrato 5 e 13 municípios no estrato 6. Não houve municípios classificados no estrato 3. Deve-se olhar com cuidado para os resultados apresentados no estrato 2 , uma vez que variações neste município podem não representar as características reais de outros municípios fora do estudo que se enquadrariam neste estrato.

O resultado da análise descritiva das coberturas e indicadores de saúde bucal está expresso nas tabelas 1 e 2 . Os valores estimados pelo modelo de regressão em relação à "cob. SB trad.”, “cob. SB/ ESF” e indicadores de saúde bucal estão expressos respectivamente nas tabelas 3 e 4 .

\section{Coberturas populacionais}

Foi constatada uma diminuição das coberturas de saúde bucal entre 2014 e 2017 na RRAS 13, principalmente à custa de queda de cobertura pelo modelo tradicional (Tabela 1). Os municípios do estrato 1 apresentaram as maiores "cob. SB" $(91 \%$ a 95\%), sendo 83\% a 91\% “cob. SB/ESF”. As menores “cob. SB" ficaram por conta dos estratos municipais 5 e 6 . Importante citar que independente dos estratos, as coberturas de ESF se mantiveram maiores do que as coberturas do modelo tradicional. Houve tendência de aumento ou manutenção nas coberturas da ESF, por outro lado houve tendência de queda das coberturas no modelo tradicional. A exceção ocorreu com municípios do estrato 6, de forma a haver manutenção da "cob. SB trad." 
TABELA 1 · Descrição dos indicadores de cobertura e saúde bucal da RRAS 13 por estrato

\begin{tabular}{|c|c|c|c|c|c|c|c|}
\hline & & \multicolumn{3}{|c|}{ Coberturas } & \multicolumn{3}{|c|}{ Indicadores de Saúde Bucal } \\
\hline & & $\begin{array}{l}\text { Cob. pop. } \\
\text { SB }\end{array}$ & $\begin{array}{l}\text { Cob. SB } \\
\text { trad. }\end{array}$ & Cob. SB/ESF & $\begin{array}{l}\text { Média } \\
\text { escov. sup. }\end{array}$ & Cob. 1 cons. & $\begin{array}{l}\text { Prop. exo/ } \\
\text { proc. }\end{array}$ \\
\hline \multirow{4}{*}{$\begin{array}{l}\text { Médias \% } \\
\text { Estrato } 1\end{array}$} & 2014 & 91,99 & 8,13 & 83,86 & 8,77 & 18,75 & 7,98 \\
\hline & 2015 & 91,93 & 8,32 & 83,61 & 9,66 & 19,23 & 5,40 \\
\hline & 2016 & 96,49 & 7,26 & 89,23 & 6,19 & 15,65 & 6,35 \\
\hline & 2017 & 95,76 & 4,71 & 91,05 & 4,18 & 11,72 & 6,88 \\
\hline \multirow{4}{*}{$\begin{array}{l}\text { Médias \% } \\
\text { Estrato } 2\end{array}$} & 2014 & 59,56 & 59,56 & 0,00 & 1,15 & 7,82 & 10,28 \\
\hline & 2015 & 87,76 & 69,94 & 17,82 & 0,95 & 9,29 & - \\
\hline & 2016 & 93,35 & 66,25 & 26,10 & - & 14,27 & 24,12 \\
\hline & 2017 & 84,25 & 58,72 & 25,53 & 4,67 & 16,26 & 25,04 \\
\hline \multirow{4}{*}{$\begin{array}{l}\text { Médias \% } \\
\text { Estrato } 4\end{array}$} & 2014 & 76,14 & 31,54 & 44,60 & 4,99 & 16,28 & 7,87 \\
\hline & 2015 & 75,41 & 30,06 & 45,35 & 4,49 & 14,33 & 8,17 \\
\hline & 2016 & 71 & 28,16 & 42,84 & 4,14 & 13,49 & 8,78 \\
\hline & 2017 & 66,99 & 25,78 & 41,21 & 4,20 & 12,85 & 8,41 \\
\hline \multirow{4}{*}{$\begin{array}{l}\text { Médias \% } \\
\text { Estrato } 5\end{array}$} & 2014 & 44,82 & 17,94 & 26,88 & 1,80 & 9,20 & 6,87 \\
\hline & 2015 & 44,42 & 16,41 & 28,01 & 1,88 & 10,53 & 6,12 \\
\hline & 2016 & 41,85 & 13,19 & 28,66 & 2,55 & 8,71 & 7,44 \\
\hline & 2017 & 42,05 & 13,35 & 28,70 & 1,88 & 10,47 & 7,45 \\
\hline \multirow{4}{*}{$\begin{array}{l}\text { Médias \% } \\
\text { Estrato } 6\end{array}$} & 2014 & 46,36 & 13,88 & 32,48 & 2,81 & 08,09 & 5,69 \\
\hline & 2015 & 49,71 & 14,52 & 35,19 & 2,12 & 7,82 & 06,07 \\
\hline & 2016 & 49,98 & 14,94 & 35,04 & 2,28 & 7,29 & 5,92 \\
\hline & 2017 & 40,98 & 14,08 & 26,90 & 1,92 & 6,54 & 6,54 \\
\hline
\end{tabular}

RRAS: rede regional de atenção à saúde; cob. pop. SB: porcentagem de cobertura populacional estimada pelas Equipes Básicas de Saúde Bucal; cob. SB/ESF: porcentagem de cobertura populacional estimada pelas equipes básicas de saúde bucal na Estratégia de saúde da família; cob. SB trad.: porcentagem de cobertura populacional estimada pelas Equipes Básicas de Saúde Bucal modelo tradicional; média escov. Sup.: média da ação coletiva de escovação dental supervisionada; cob. 1 cons.: cobertura de primeira consulta odontológica programática; prop. exo/proc.: proporção de exodontia em relação aos procedimentos. 
(13\% em 2014 e 14\% em 2017) em detrimento de queda da "cob. SB/ESF” (32\% em 2014 para 26\% em 2017, alcançando em 2015 e 2016 35\%) (Tabela 1).

Indicadores de saúde bucal

No âmbito da RRAS 13, ao longo dos anos 2014 a 2017, houve diminuição dos indicadores "média escov. sup." e “cob. 1 cons”. Constatou-se ainda o aumento do indicador "prop. exo/proc", ou seja, piora dos três indicadores de saúde bucal (Tabela 2).

Os municípios dos estratos 5 e 6 apresentaram as menores "médias escov. Sup". (entre 1,80 e 2,81\% em toda série histórica), com tendência de manutenção do valor dos indicadores durante os anos analisados. Já os estratos 1, 2 e 4 apresentaram no ano de 2017 a "média escov. sup." em 4,20; 4,67 e 4,18\%, respectivamente, havendo no estrato 1 tendência de queda $(8,77 \%$ em 2014$)$, no estrato 2 tendência de aumento (1,15\% em 2014) e manutenção no estrato 4 (4,99\% em 2014).

TABELA 2 · Descrição dos indicadores de cobertura e saúde bucal da RRAS 13

Coberturas / Indicadores de Saúde Bucal Média \% Todos os municípios

\begin{tabular}{lcccc}
\hline & $\mathbf{2 0 1 4}$ & $\mathbf{2 0 1 5}$ & $\mathbf{2 0 1 6}$ & $\mathbf{2 0 1 7}$ \\
\hline Cob. Pop. SB & 62,19 & 62,54 & 60,07 & 56,83 \\
\hline Cob. SB. Trad & 23,36 & 22,41 & 20,46 & 19,05 \\
\hline Cob. SB/ESF & 39,26 & 40,54 & 40,05 & 38,26 \\
\hline Média Escov. Sup. & 3,90 & 3,82 & 3,79 & 3,37 \\
\hline Cob. 1 Cons. & 12,03 & 12,24 & 11,88 & 11,57 \\
\hline Prop. Exo/Proc. & 7,74 & 6,44 & 10,52 & 10,86 \\
\hline
\end{tabular}

RRAS: rede regional de atenção à saúde; cob. pop. SB: porcentagem de cobertura populacional estimada pelas Equipes Básicas de Saúde Bucal; cob. SB/ESF: porcentagem de cobertura populacional estimada pelas equipes básicas de saúde bucal na Estratégia de saúde da família; cob. SB trad.: porcentagem de cobertura populacional estimada pelas Equipes Básicas de Saúde Bucal modelo tradicional; média escov. Sup.: média da ação coletiva de escovação dental supervisionada; cob. 1 cons.: cobertura de primeira consulta odontológica programática; prop. exo/proc.: proporção de exodontia em relação aos procedimentos. 
Semelhantemente os municípios dos estratos 5 e 6 apresentaram as menores médias de "cob. 1 cons.”, havendo tendência de manutenção destes indicadores nos municípios do estrato 5 (9\% em 2014 para 10\% em 2017) e tendência de diminuição no estrato 6 (8\% em 2014 para 6\% em 2017). Maiores valores para o indicador foram encontrados nos municípios dos estrados 1, 2 e 4.

TABELA 3 - Valores estimados pelo modelo de regressão em relação à "cob SB trad" e indicadores de saúde bucal

\begin{tabular}{llllll}
\hline Efeito & Média P & DP & Int Credib (95\%) & \\
\hline IDH-M como preditor & & & & & \\
\hline Média Escov Sup & 0,0078 & 0,0005 & 0,0069 & a & 0,0087 \\
\hline Cob 1 Cons & $-0,0016$ & 0,0001 & $-0,0019$ & a & $-0,0014$ \\
\hline Prop Exo/Proc & 0,0212 & 0,0007 & 0,0199 & a & 0,0226 \\
\hline
\end{tabular}

IDH-E como preditor

\begin{tabular}{lllllc}
\hline Média Escov Sup & 0,0084 & 0,0005 & 0,0075 & a & 0,0093 \\
\hline Cob 1 Cons & $-0,0014$ & 0,0001 & $-0,0017$ & a & $-0,0012$ \\
\hline Prop Exo/Proc & 0,0249 & 0,0007 & 0,0236 & a & 0,0263 \\
\hline
\end{tabular}

IDH-R como preditor

\begin{tabular}{lccccc}
\hline Média Escov Sup & 0,0080 & 0,0005 & 0,0071 & a & 0,0089 \\
\hline Cob 1 Cons & $-0,0016$ & 0,0001 & $-0,0018$ & a & $-0,0013$ \\
\hline Prop Exo/Proc & 0,0237 & 0,0007 & 0,0224 & a & 0,0250 \\
\hline
\end{tabular}

Gini como preditor

\begin{tabular}{lccccc}
\hline Média Escov Sup & 0,0087 & 0,0005 & 0,0078 & a & 0,0096 \\
\hline Cob 1 Cons & $-0,0014$ & 0,0001 & $-0,0017$ & a & $-0,0011$ \\
\hline Prop Exo/Proc & 0,0228 & 0,0006 & 0,0216 & a & 0,0241 \\
\hline
\end{tabular}

Cob SB trad: cobertura populacional estimada pelas equipes básicas de saúde bucal no modelo tradicional; média P: média Posteriori; DP: desvio padrão; int. credib: intervalo de credibilidade; IDH-M: Índice de Desenvolvimento Humano Municipal; IDH-E: componente educação do IDH; IDH-R: componente Renda do IDH; média escov. Sup.: média da ação coletiva de escovação dental supervisionada; cob. 1 cons.: cobertura de primeira consulta odontológica programática; prop. exo/proc.: proporção de exodontia em relação aos procedimentos. 
As tendências foram de queda para os estratos 1 e $4(18,75 \%$ e $16,28 \%$ em 2014 para 11,72\% e 12,82\% em 2017 respectivamente).

Em relação ao indicador “prop. exo/proc.”, os municípios apresentaram valores semelhantes, independentemente dos estratos, porém municípios do estrato 6 apresentaram valores menores. Houve leve aumento com menos de 1 ponto percentual nos estratos 4,5 e $6(7,87 ; 6,87$ e 5,69\% em 2014 para 8,$41 ; 7,45$ e $6,54 \%$ em 2017). No estrato 1 a tendência foi de queda (7,98\% em 2014 para $6,88 \%$ em 2017). No município do estrato 2 observou-se valores muito elevados (10,28\% em 2014 para 25,04\% em 2017, não havendo o dado do ano de 2015) (Tabela 1).

Relação entre coberturas de saúde bucal e indicadores de saúde bucal

Assumindo a análise proposta, para que haja evidência de relação estatística entre as variáveis, o numeral 0 não pode estar incluso no Intervalo de Credibilidade com 95\% de confiança, interpretação análoga ao intervalo de confiança utilizado em inferência estatística clássica. A Média Posteriori (Média P) mostra se a associação é positiva (proporcional) ou negativa (inversamente proporcional), mostra também a intensidade desta associação, sendo quando maior o numeral, maior a intensidade da associação.

As correlações ocorreram semelhantemente independente do preditor. O indicador 0 "média escov. sup." apresentou relação estatística positiva com “cob. SB/ESF” (Média P = 0.0030) e ”cob. SB trad." (Média $\mathrm{P}=0.0078)$. Já o indicador “cob. 1 cons.” se comportou de forma inversamente proporcional com a "cob. SB trad." (Média $\mathrm{P}=-0.0016)$ e diretamente proporcional com "cob. SB/ESF" (Média $\mathrm{P}=0.0039$ ). O indicador "prop exo/proc." mostrou evidente relação estatística positiva com "cob. SB trad.” (Média $\mathrm{P}=0.0212$ ) e negativa com “cob. SB/ESF” (Média $\mathrm{P}=-0.0173)$ (Tabelas 3 e 4). 
TABELA 4 · Valores estimados pelo modelo de regressão em relação à Cob SB/ESF e indicadores de saúde bucal

\begin{tabular}{llllll}
\hline Efeito & Média P & DP & \multicolumn{2}{l}{ Int Credib (95\%) } \\
\hline IDH-M como preditor & & & & & \\
\hline Média Escov Sup & 0,0030 & 0,0003 & 0,0023 & a & 0,0036 \\
\hline Cob 1 Cons & 0,0039 & 0,0002 & 0,0036 & a & 0,0043 \\
\hline Prop Exo/Proc & $-0,0173$ & 0,0008 & $-0,0189$ & a & $-0,0158$ \\
\hline
\end{tabular}

IDH-E como preditor

\begin{tabular}{lccccc}
\hline Média Escov Sup & 0,0035 & 0,0003 & 0,0029 & a & 0,0042 \\
\hline Cob 1 Cons & 0,0047 & 0,0002 & 0,0043 & a & 0,0050 \\
\hline Prop Exo/Proc & $-0,0098$ & 0,0008 & $-0,0114$ & a & $-0,0083$
\end{tabular}

IDH-R como preditor

\begin{tabular}{lccccc}
\hline Média Escov Sup & 0,0039 & 0,0003 & 0,0032 & a & 0,0045 \\
\hline Cob 1 Cons & 0,0030 & 0,0001 & 0,0028 & a & 0,0033 \\
\hline Prop Exo/Proc & $-0,0229$ & 0,0008 & $-0,0244$ & a & $-0,0213$ \\
\hline
\end{tabular}

Gini como preditor

\begin{tabular}{lccccc}
\hline Média Escov Sup & 0,0032 & 0,0003 & 0,0025 & a & 0,0038 \\
\hline Cob 1 Cons & 0,0043 & 0,0002 & 0,0040 & a & 0,0046 \\
\hline Prop Exo/Proc & $-0,0141$ & 0,0008 & $-0,0157$ & a & $-0,0126$ \\
\hline
\end{tabular}

Cob SB/ESF: cobertura populacional estimada pelas equipes básicas de saúde bucal na Estratégia de Saúde da Família; média P: média Posteriori; DP: desvio padrão; int. credib: intervalo de credibilidade; IDH-M: Índice de Desenvolvimento Humano Municipal; IDH-E: componente educação do IDH; IDH-R: componente Renda do IDH; média escov. Sup.: média da ação coletiva de escovação dental supervisionada; cob. 1 cons.: cobertura de primeira consulta odontológica programática; prop. exo/proc.: proporção de exodontia em relação aos procedimentos.

\section{Discussão}

Este trabalho permitiu observar que os municípios com maiores coberturas pela ESF apresentaram um melhor desempenho nos indicadores de saúde bucal, porém a correlação encontrada, mesmo que significante foi fraca. Adicionalmente observamos que ao longo do período estudado houve uma diminuição nas coberturas de saúde bucal principalmente às custas da cobertura de saúde bucal tradicional e piora dos indicadores de saúde bucal. 
Uma limitação dos trabalhos que utilizam dados secundários, incluindo este, é a possível discordância nas ações efetivamente realizadas e ações informadas pelo município ${ }^{14,15}$, ainda assim, dados secundários são amplamente utilizados na literatura, pois se acredita que tais informações expressem a situação sanitária de uma população. Outra limitação foi a impossibilidade de inclusão de outros indicadores de saúde bucal devido à ausência e desatualização de dados nos sistemas de informações.

Diversas são as metodologias adotadas para verificar se as Equipes de Saúde Bucal da ESF (ESB/ESF) proporcionam à população coberta melhores indicadores, acesso e condição de saúde quando comparado à população coberta por equipes de saúde bucal fora da ESF (ESB/Tradicional). Um trabalho comparou indicadores de saúde bucal entre Unidades de Saúde da Família e Tradicionais com equipes de saúde bucal através dos dados produzidos pelas unidades, sendo todas pertencentes a um mesmo município ${ }^{16}$. Outro trabalho comparou dados de utilização de serviços e procura por assistência em distritos de um município os quais alguns eram cobertos por ESB/ESF e outros por ESB/ $\operatorname{Trad}^{17}$. Thurow et al. $^{18}$ (2015) avaliaram os procedimentos odontológicos realizados pelos dentistas alocados em Unidades de Saúde da Família ou Tradicionais, também no contexto de uma única cidade. A avaliação no contexto de áreas, distritos ou unidades pertencentes a um único município, como nos trabalhos citados acima, requer que a coleta de dados seja feita através de entrevista a indivíduos ou disponibilização da produção pelas Unidades de Saúde uma vez que estas informações não estão disponíveis no DATASUS.

Estudos ${ }^{19,20}$ utilizaram setores censitários para emparelhamento das áreas cobertas e não cobertas pelas equipes de saúde bucal da ESF com base em critérios socioeconômicos. Todos fizeram a coleta de dados por entrevista estruturada. Geralmente, por se tratar de uma metodologia que requer mais tempo e recursos, o universo de estudo se limita a um único município, porém 
existe um trabalho que avalia mais municípios ${ }^{21}$. A vantagem de emparelhamento das áreas cobertas e não cobertas pela ESF é o controle das amostras em relação às características sociodemográficas de cada unidade. Uma alternativa utilizada no atual trabalho e também por Goldbaum et al. ${ }^{17}$ (2005) é considerar aspectos socioeconômicos adicionando-os na análise estatística como variáveis de confusão.

O presente trabalho propõe uma metodologia reprodutível, sem altos custos e facilmente reproduzida em outros contextos. Grande parte das publicações que comparam indicadores de saúde bucal em áreas cobertas e não cobertas pelas equipes de saúde bucal na ESF se concentram entre os anos de 2000 a 2015, sendo difícil encontrar trabalhos atuais sobre o tema. Foram inclusos 90 municípios como universo de estudo e avaliação em série histórica uma vez que a maioria dos trabalhos com objetivo semelhante se limitam a fazer uma avaliação transversal no contexto de em um ou poucos municípios, o que dificulta a aplicação dos resultados de forma mais ampla.

A queda da cobertura de saúde bucal pode ser explicada pelo atual cenário político e econômico do nosso país, com o contingenciamento de gastos na área da saúde e educação ${ }^{22}$ e necessidade de reformas. Os investimentos em saúde considerados menos prioritários são reduzidos, logo o acesso aos cuidados médicos é anteposto à saúde bucal. Ainda sim nota-se o esforço de manutenção da “cob. SB/ESF”.

As maiores “cob. SB/ESF” estão nos municípios dos estratos 1 e 4 , principalmente no estrato 1 , o que pode ser explicado pela necessidade de poucas equipes de Saúde da Família para cobrir toda a população. Os municípios dos estratos 5 e 6 possuem menores coberturas de saúde bucal na ESF. Em concordância com esses achados, a literatura mostra correlação negativa entre IDH e Proporção de população coberta pelo Programa de Saúde da Família $^{3}$, tal correlação caminha no sentido pró-equidade. 
Os indicadores de saúde bucal apresentaram padrões relacionados aos estratos municipais, como o indicado: A "prop. exo/ proc." encontrada foi semelhante em todos os estratos, porém municípios dos estratos 5 e 6 apresentaram menores valores, o que pode ser interpretado como a prática de uma odontologia menos mutiladora. Em concordância com os resultados encontrados Pimentel et al. ${ }^{23}$ (2014) constataram que municípios de grande porte apresentaram menores valores para proporção de exodontias. Fernandes e Peres ${ }^{15}$ (2005) também encontraram associação entre municípios com menor IDH e maior proporção de exodontias executadas. Outro trabalho confirma o impacto do IDH, GINI e presença de Centros de Especialidades Odontológicas (CEOs) na quantidade de extrações dentárias realizadas ${ }^{24}$. Acredita-se que esse comportamento ocorra devido a uma maior estruturação dos serviços de saúde, presença de sistemas de referência e consequentemente acesso a centros especializados (CEO).

Municípios de menor porte (estratos 1, 2 e 4) apresentaram melhor desempenho nos indicadores "média escov. sup." e “cob. 1 cons". Uma hipótese seria a de que municípios de menor porte apresentam maior facilidade na gestão dos serviços de saúde e maior possibilidade de impacto de políticas sobre os indicadores visando ampliação do acesso aos serviços e aos programas preventivos. Outra explicação seria a maior “cob. SB/ESF” nestes municípios, porém essa relação será discutida adiante.

As médias de “escov. sup.” nos municípios da RRAS 13 variaram de 3,15 a 3,17\%, o que é considerado baixo quando comparado a meta de 5\% estipulada pela Portaria n 91/GM, de 10 de janeiro de 2007 . As médias de "cob. 1 cons." variaram entre $11,12 \%$ a $12,85 \%$, também considerado baixo quando comparadas à meta de $30 \%$ estipulada pela mesma portaria.

Este estudo utilizou os IDH Municipal e seus componentes renda e educação, e o Índice de Gini como preditores no modelo estatístico com objetivo de não ocorrer em viés metodológico, 
uma vez que aspectos socioeconômicos impactam diretamente nos indicadores analisados. Rocha e Goes ${ }^{20}$ (2008) em seu estudo verificaram que aqueles que residiam em áreas não cobertas pela ESF obtiveram 1,5 vez mais chance de ter acesso aos serviços de saúde bucal quando comparados aos que residiam em áreas cobertas pela ESF. No entanto, essa probabilidade diminuiu, perdendo a sua significância após o resultado ser ajustado para sexo, idade, renda individual, escolaridade e autopercepção de saúde.

A relação positiva encontrada entre a "cob. SB trad." e a "média escov. sup." pode estar relacionada a perpetuação do modelo incremental, com ênfase em prevenção em escolares e exclusão de parcela significante da população ${ }^{25}$, sendo que esta prática pode estar sendo reproduzida mesmo pelas equipes de saúde bucal na ESF $^{19,26}$. Para melhor elucidação seria necessário avaliar quais grupos populacionais estão tendo acesso a estas ações. Outro fator que justifica o bom desempenho deste indicador relacionado a Cob. SB Trad. é o fato de que a "média escov. sup." é cobrada dos municípios desde 2006 com o Pacto pela Saúde.

A relação positiva da “cob. SB/ESF." e "média escov. sup.” pode ser justificada pelos mesmos motivos, além de que neste modelo há ênfase na realização de ações preventivas como a escovação dental supervisionada.

O aumento da “prop. exo/proc.” relacionado ao modelo assistencial tradicional já foi descrito na literatura ${ }^{14}$, essa variação geralmente está associada a um grande número de exodontias realizadas, caracterizando um modelo curativista mutilatório.

O pior desempenho do indicador "cob. 1 cons." relacionado à “cob. SB trad.” reflete a ineficiência deste modelo em proporcionar acesso. A ausência de população adscrita levando ao atendimento da demanda espontânea, baseada no controle de condições agudas, são fatores que dificultam o acesso àqueles que desejam iniciar o tratamento odontológico. 
A análise estatística mostrou que a "cob SB/ESF" tem evidência de impacto positivo nos indicadores "média escov. sup.", "cob. 1 cons." e impacto negativo na "prop. exo/proc.", ou seja, a presença de equipes de saúde bucal na ESF se relaciona com maior execução de ações preventivas, maior acesso e um modelo com predominância na realização de mais procedimentos preventivos e curativos e menor número de procedimentos cirúrgicos, sendo que estes achados estão em sintonia com a proposta da ESF como reorientadora da Atenção Básica.

Na literatura, porém, não existe consenso sobre o assunto. Thurow et al. ${ }^{18}$ (2015) comparando unidades com equipes de saúde bucal (ESB) na ESF e aquelas com modelo tradicional de odontologia (MTO), não observaram diferenças estatisticamente significativas nas médias dos indicadores de primeira consulta odontológica programática, tratamento concluído, atendimento de urgência, atendimento a gestante, encaminhamento para atenção secundária em saúde bucal e alteração de mucosa. Houve diferença, sim, com melhor resultado nas ESB/ESF na média da razão de tratamentos concluídos/primeiras consultas, média de escovação dental supervisionada e média de procedimentos coletivos. Barros e Chaves ${ }^{14}$ (2003) observaram um aumento geral da produção ambulatorial odontológica no município com $\mathrm{ESB} / \mathrm{ESF}$ e com ESB/tradicional, porém o município com ESB/ESF apresentou maior ênfase nos procedimentos coletivos quando comparado ao município com ESB/tradicional que por sua vez apresentou um modelo mais centrado em ações curativas individuais.

Outros autores, porém, encontraram resultados contrários à superioridade da ESF em promover melhores indicadores. Magri et al. ${ }^{16}$ (2016) compararam indicadores de saúde de unidades USF e UBS. Foi encontrada significância estatística na comparação USF/UBS apenas para o indicador "relação restauração/ extração” e “relação 1' consulta/urgência”, sendo que os melhores resultados foram observados no modelo assistencial UBS. 
Não houve diferença estatística para os indicadores "ênfase em prevenção modificado", que compara a proporção de procedimentos preventivos versus curativos, "indicador de atrição", dado pelo valor percentual de quanto da população teve acesso a tratamento odontológico e efetivamente realizou todo o tratamento, e "indicador de rendimento", que expressa a capacidade produtiva da hora do Cirurgião-Dentista (CD) na assistência. Rocha e Goes ${ }^{20}$ (2008) como citado anteriormente, constataram não haver diferença no acesso aos serviços de saúde (consulta ao dentista nos últimos 2 anos) entre ESB/ESF e ESB/UBS.

Acredita-se que essas discordâncias ocorram devido às diferentes formas de implementação, estruturação, apoio da gestão municipal, características regionais e socioeconômicas dos diferentes municípios, conforme já descrito por Souza e Roncalli ${ }^{5}$ (2007). Nesse sentido é importante a implantação de uma cultura avaliativa onde os gestores e profissionais do SUS acompanhem os indicadores e o processo de trabalho de suas equipes, buscando mudança do modelo assistencial. Estudos futuros devem esclarecer quais são os nós críticos para uma reorientação efetiva do modelo assistencial, uma vez que ESB/ESF podem estar trabalhando na lógica do modelo tradicional, o que explicaria a correlação fraca encontrada entre a “cob. pop. SB/ESF” e melhora dos indicadores.

\section{Conclusão}

Pode-se concluir que nos municípios da RRAS 13 as coberturas de saúde bucal na ESF já superam as coberturas de outros modelos assistenciais; as coberturas populacionais e os indicadores de saúde bucal apresentaram padrões relacionados aos estratos municipais, ou seja, aspectos socioeconômicos e populacionais foram determinantes em seu comportamento; os estratos propostos pelo PMAQ-AB se mostraram ferramentas válidas na categorização dos municípios, proporcionando interpretação dos dados no contexto de municípios com características 
semelhantes; os indicadores de saúde bucal encontrados nos municípios em estudo são considerados insatisfatórios quando comparados aos valores preconizados pela portaria n 91/GM, de 10 de janeiro de 2007; as equipes de saúde bucal na ESF e a ampliação da cobertura se correlacionaram com uma melhora dos indicadores de saúde bucal, porém a correlação encontrada, mesmo que significante foi fraca.

\section{Referências}

1 - Rede Interagencial de Informação para a Saúde. Indicadores básicos para saúde no brasil: conceitos e aplicações. In: Rede Interagencial de informação para a saúde. Indicadores de saúde e a ripsa. Brasília: Ed. Organização Pan-Americana da Saúde; 2008. p. 11-18.

2 - Departamento de Atenção Básica. Informação e Gestão da Atenção Básica 2020.[internet]; 2020 [acesso em 23 jan 2020] Brasília: Ministério da Saúde. Disponível em: https://egestorab.saude.gov.br/

3 - Fernandes JKB, Pinho JRO, Queiroz RCS, Thomaz EBAF. Avaliação dos indicadores de saúde bucal no Brasil: tendência evolutiva pró-equidade? Cad. Saúde Pública. 2016; 32(2). doi: https://doi. org/10.1590/0102-311X00021115

4 - Roncalli AG, Lima KC. Impacto do Programa Saúde da Família sobre indicadores de saúde da criança em municípios de grande porte da região Nordeste do Brasil. Cienc e Saúde Colet. 2006; 11(3): 713-24. doi: http://dx.doi.org/10.1590/S1413-81232006000300018

5 - Souza TMS, Roncalli AG. Saúde bucal no Programa Saúde da Família: uma avaliação do modelo assistencial. Cad Saúde Pública. 2007; 3(11): 2727-39. doi: http://dx.doi.org/10.1590/S0102-311X2007001100020

6 - Departamento de Atenção Básica. Programa de melhoria do acesso e da qualidade da atenção básica - PMAQ-AB [Internet]. [citado em 24 Jan 2019]. Disponível em: http://dab.saude.gov.br/sistemas/pmaq/estratos_ para_certificacao.php

7 - Programa das Nações Unidas para o Desenvolvimento. Atlas do Desenvolvimento Humano no Brasil [Internet]. [citado em 27 Jan 2019]. Disponível em: http://www.atlasbrasil.org.br/2013/

8 - Departamento de Atenção Básica. Manual Instrutivo para as Equipes de Atenção Básica e NASF [Internet]. 2017 [citado em 17 Abr 2019]. Disponível em: http://189.28.128.100/dab/docs/portaldab/documentos/ Manual_Instrutivo_3_Ciclo_PMAQ.pdf

9 - Ministério da Saúde, Secretaria de Gestão Estratégica e Participativa, Departamento de Articulação Interfederativa. Caderno de Diretrizes [Internet]. 2nd ed. 2014; 1: 154 [citado em 15 Mar 2019]. Disponível em: 
http://189.28.128.100/sispacto/CadernoDiretrizes2013_2015.pdf

10 - Blangiardo M, Cameletti M. Spatial and spatio-temporal Bayesian models with R-INLA. Nova Iorque: John Wiley \& Sons; 2015.

11 - Besag J, York J, Mollie A. Bayesian image restoration, with two applications in spatial statistics. Annals of the Institute of Statistical Mathematics. 1991; 43:1-59. doi: https://doi.org/10.1007/BF00116466

12 - Rue H, Martino S, Chopin N. Approximate Bayesian inference for latent Gaussian models using integrated nested Laplace approximations. Journal of the Royal Statistical Society. 2009; 71(2):319392. doi: https://doi.org/10.1111/j.1467-9868.2008.00700.x

13 - Lindgren F, Rue H, Lindstrom J. An explicit link between Gaussian fields and Gaussian Markov random fields: The SPDE approach. Journal of the Royal Statistical Society. 2011; 73(4):423-498. doi: https:// doi.org/10.1111/j.1467-9868.2011.00777.x

14 - Barros SG, Chaves SCL. A utilização do Sistema de Informações Ambulatoriais (SIA-SUS) como instrumento para caracterização das ações de saúde bucal. Epidemiol. Serv. Saúde. 2003; 12(1): 41-51. doi: http://dx.doi.org/10.5123/S1679-49742003000100005

15 - Fernandes LS, Peres MA. Associação entre atenção básica em saúde bucal e indicadores socioeconômicos municipais. Rev Saúde Púb. 2005; 39(6): 930-6. DOI: https://doi.org/10.1590/S0034-89102005000600010

16 - Magri LV, Aciole GG, Salomão FGD, Tagliaferro EPS, Ribeiro LG. Estudo comparativo de indicadores de saúde bucal em município do estado de São Paulo. Saúde Debate. 2016; 40(108): 144-55. doi: https:// doi.org/10.1590/0103-1104-20161080012

17 - Goldbaum M, Gianini RJ, Novaes HMD, César CLG. Utilização de serviços de saúde em áreas cobertas pelo programa saúde da família (Qualis) no Município de São Paulo. Rev Saúde Púb. 2005; 39(1):90-9. doi: https://doi.org/10.1590/S0034-89102005000100012

18 - Thurow LL, Castilhos ED, Costa JSD. Comparação das práticas odontológicas segundo modelos de atendimento: tradicional e da Saúde da Família, Pelotas-RS, 2012-2013. Epidemiol Serv Saúde. 2015; 24(3):545-50. doi: https://doi.org/10.5123/S1679-49742015000300021

19 - Pereira CRS, Patrício AAR, Araújo FAC, Lucena EES, Lima KC, Roncalli AG. Impacto da Estratégia Saúde da Família com equipe de saúde bucal sobre a utilização de serviços odontológicos. Cad Saúde Púb. 2009; 25(5):985-96. doi: https://doi.org/10.1590/S0102311X2009000500005

20 - Rocha RACP, Goes PSA. Comparação do acesso aos serviços de saúde bucal em áreas cobertas e não cobertas pela Estratégia Saúde da Família em Campina Grande, Paraíba, Brasil. Cad Saúde Púb. 2008; 24(12): 2871-80. doi: https://doi.org/10.1590/S0102-311X2008001200016

21 - Pereira CRS, Roncalli AG, Cangussu MCT, Noro LRA, Patrício AAR, 
Lima KC. Impacto da Estratégia Saúde da Família sobre indicadores de saúde bucal: análise em municípios do Nordeste brasileiro com mais de 100 mil habitantes. Cad Saúde Púb. 2012; 28(3): 449-62. doi: https://doi. org/10.1590/S0102-311X2012000300005

22 - Probst LF, Pucca Junior GA, Pereira AC, Carli ADD. Impacto das crises financeiras sobre os indicadores de saúde bucal: revisão integrativa da literatura. Ciênc saúde colet. 2019; 24(12): 4437-48. doi: https://doi. org/10.1590/1413-812320182412.23132019

23 - Pimentel FC, Albuquerque PC, Martelli PJL, Acioli RML, Souza WV. Analysis of oral health indicators of Pernambuco: performance of cities according to size population, population enrolled in the Information System for Primary Care and proportion in the Family Health Strategy. Cad Saúde Colet. 2014; 22(1): 54-61. doi: https://doi.org/10.1590/1414462X201400010009

24 - Silva DRB, Lucena CDRX, Cruz DF, Figueiredo N, Goes PSA, Lucena EHG. Análise do indicador de extração dentária a partir do contexto municipal. Revista Família, Ciclos de Vida e Saúde no Contexto Social. 2018; 6(2): 220-7. doi: https://doi.org/10.18554/refacs.v6i2.2819

25 - Nickel DA, Lima FG, Silva BB. Modelos assistenciais em saúde bucal no Brasil. Cad Saúde Púb. 2008; 24(2):241-6. doi: https://doi.org/10.1590/ S0102-311X2008000200002

26 - Almeida GCM, Ferreira MÂF. Saúde bucal no contexto do Programa Saúde da Família: práticas de prevenção orientadas ao indivíduo e ao coletivo. Cad Saúde Púb.2008; 24(9): 2131-40. doi: https:/doi. org/10.1590/S0102-311X2008000900019 


\title{
The impact of the family health strategy on oral health indicators
}

\begin{abstract}
Aim: To assess the impact of Family Health Strategy coverage on oral health indicators in the 90 municipalities of RRAS 13, taking into account socioeconomic aspects, in an analysis of a historical series between the years 2014 and 2017. Material and method: through the Ambulatory Information System, eGestor and DATASUS platforms, the population coverage by the health services variables were obtained and the oral health indicators were calculated. Socioeconomic aspects were considered using weighting by the municipal HDI, its components of income, education and Gini index. For the analysis of the data, a descriptive analysis was performed and later a Bayesian temporal space model was proposed using a spatial Poisson model with a random BYM effect. The social indicators entered as predictors in the model, which was implemented with computational support from the $\mathrm{R}$ software and used Laplace approximation methods. Results: they pointed out that there is evidence of a statistical relationship of oral health coverage in the Family Health Strategy with improvement of the indicators "proportion of tooth extraction in relation to the procedures" and "coverage of the first programmatic dental consultation". The oral health coverage in the traditional model, on the other hand, was statistically related to the worsening of these two indicators. Conclusion: Increased coverage by oral health teams in the FHS was associated with improved oral health indicators assessed in this study, when compared to the traditional model.
\end{abstract}

KEYWORDS: Health Status Indicators; Health Impact Assessment; Primary Health Care; Family Health Strategy; Oral Health.

\section{Como citar este artigo}

Reis TC, Fumagalli IHT, Lago LPM, Mestriner SF, Ramos MM, Mestriner Júnior W. 0 impacto da estratégia saúde da família nos indicadores de saúde bucal. Rev Odontol Bras Central 2021; 30(89): 300-322. DOI: 10.36065/robrac.v30i89.1485 